AeDA/DGAKI Notes

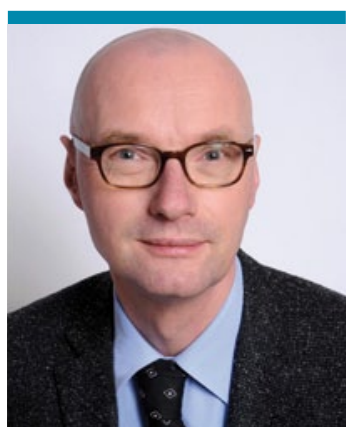

Prof. Dr. Harald

Renz,

Institut für

Laboratori-

umsmedizin,

Uniklinik

Gießen und

Marburg,

Präsident

der DGAKI

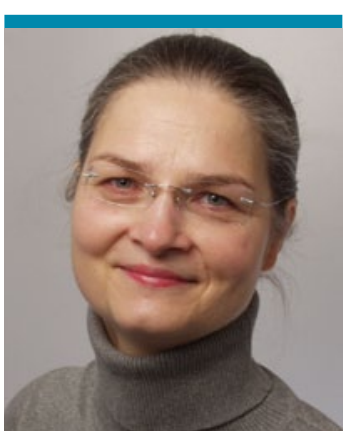

PD Dr.

Kirsten

Jung,

Praxis für

Dermatolo-

gie und Im-

munologie,

Erfurt,

Präsidentin

des AeDA

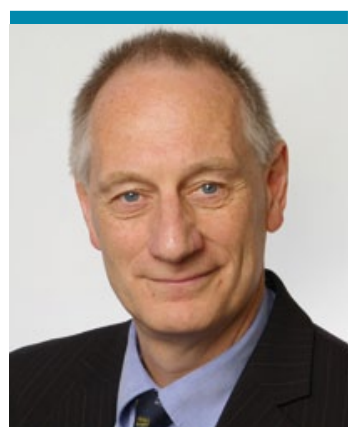

Prof. Dr.

Albrecht

Bufe,

Abteilung für

experimen-

telle Pneu-

mologie,

Uniklinik

Bergmanns-

heil, Bochum,

Präsident der

GPA

\title{
Allergie und Umwelt in Bochum
}

m Namen der Deutschen Gesellschaft für Allergologie und klinische Immunologie (DGAKI), der Gesellschaft für Pädiatrische Allergologie und Umweltmedizin (GPA) und des Ärzteverbandes Deutscher Allergologen (AEDA) möchten wir Sie auf das Herzlichste nach Bochum einladen!

Der Deutsche Allergiekongress wird diesjährig zum achten Mal gemeinsam durchgeführt und hat sich damit als feste Marke im jährlichen Reigen allergologischer Fort- und Weiterbildungsveranstaltungen etabliert. Im Rahmen der turnusmäßig wechselnden Präsidentschaft wird der diesjährige Kongress von der DGAKI ausgerichtet und es ist uns eine besondere Freude, dass die Doppelspitze aus Prof. Dr. Eckard Hamelmann, Bochumer Kinderklinik, und Prof. Dr. Monika Raulf-Heimsoth, Institut für Prävention und Arbeitsmedizin der Deutschen Gesetzlichen Unfallversicherung (DGUV), diesen Kongress organisiert.
Der Allergiekongress in Bochum bedeutet allergologische Fort- und Weiterbildung auf höchstem Niveau im Herzen des Ruhrgebietes. Damit in Zusammenhang steht auch das Leitthema „Allergie und Umwelt“ - entsprechend der gewachsenen wissenschaftlich-akademischen Tradition der Region. Somit wird ein besonderes Augenmerk auf die Einflüsse durch Klima, Staub und Feinstaub sowie Innenraumbelastungen und Beruf gelegt.

Darüber hinaus bietet der Deutsche Allergiekongress ein jährliches Update der gesamten Allergologie. Der aktuelle Kenntnisstand kann dieses Jahr auch erstmalig zum Nachlesen mit nach Hause genommen werden - in Form eines Syllabus. Das Spektrum der allergologischen Fortbildung wird von der (Epi-) Genetik, Prävention, Diagnostik bis hin zur Therapie an allen relevanten Effektororganen reichen. Neben den etablierten Elementen einer solchen großen Tagung, wie den (Plenar)Vorträgen und
Symposien, wird es dieses Jahr erstmals auch neue attraktive Formate geben, wie "AllergoActiv" mit Workshop/Seminarcharakter, „AllergoArena“ als spannender Pro-und-Kontra-Schlagabtausch zu aktuellen und kontroversen Themen aus Praxis und Wissenschaft, den „AllergoCases" mit Fällen aus der Praxis sowie den „AllergoCompact“-Veranstaltungen als Fortbildungssymposien der besonderen Art. Eine weitere Besonderheit werden die „AllergoForen“ darstellen, Symposien mit ausgewählten Geschwisterfachgesellschaften, um über den eigenen Tellerrand hinauszuschauen und allergologische Problemfelder aus dem Blickfeld unserer Fachgesellschaften zu beleuchten.

Die Ausrichter haben sich auch dieses Jahr besondere Mühe gegeben, ein attraktives Rahmenprogramm aufzulegen, das als facettenreiches und buntes Programm uns als Kongressteilnehmern „Lust“ auf das Ruhrgebiet machen soll und wird.

Wir sind sicher, dass auch der diesjährige 8. Deutsche Allergiekongress somit zu einem großen Erfolg sowohl in wissenschaftlicher als auch in gesellschaftlicher und persönlicher Hinsicht werden wird. Willkommen in Bochum!
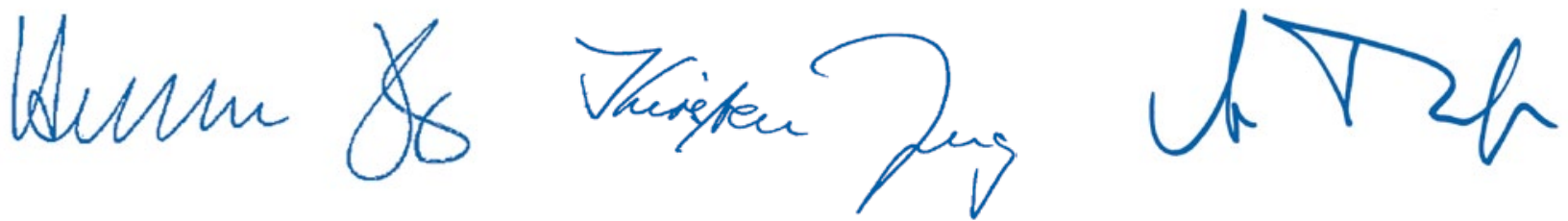